\title{
The Spectrum of Histomorphological Changes and Pathological Tumor Response following Preoperative Oral Metronomic Chemotherapy in Oral Squamous Cell Carcinoma
}

Sithara Aravind ${ }^{1}$ Jasmin Jose ${ }^{1}$ Praveen Kumar Shenoy ${ }^{2}$ Manuprasad Avaronnan ${ }^{2}$ Sajith Babu Thavarool ${ }^{3}$ Sangeetha K. Nayanar ${ }^{1}$

1 Division of Oncopathology, Malabar Cancer Centre, Thalassery, Kerala, India

2 Division of Medical Oncology, Malabar Cancer Centre, Thalassery, Kerala, India

${ }^{3}$ Division of Surgical Oncology, Malabar Cancer Centre, Thalassery,

Address for correspondence Sithara Aravind, MD, Division of Oncopathology, Malabar Cancer Centre, Thalassery -670103, Kerala, India (e-mail: sitharaaravind@gmail.com).

Kerala, India

South Asian J Cancer 2022;11(2):146-151.

\section{Abstract}

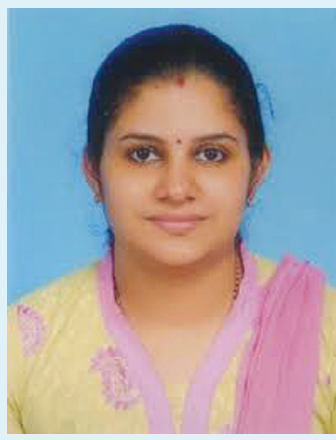

Sithara Aravind

Keywords

- histomorphological changes

- oral metronomic chemotherapy

- oral squamous cell carcinoma

- pathologic tumor response
Background Oral metronomic chemotherapy (OMCT) represents an emerging concept in cancer treatment involving frequent administration of chemotherapeutic drugs at doses below maximum tolerated doses and with no prolonged drug-free break. OMCT is being tried preoperatively in developing nations with constrained resources to prevent disease progression during the waiting period from diagnosis to surgery (bridge OMCT). The aim of the present study was to assess the spectrum of histomorphological changes and pathological tumor response following bridge OMCT in oral squamous cell carcinoma (OSCC) and to propose a new pathological response scoring system.

Materials and Methods A retrospective single-center study comprised of tissue sections of tumor proper and metastatic lymph nodes of 50 , locally advanced OSCC patients treated with bridge $\mathrm{OMCT}$, and had completed definitive surgery were analyzed. The present study evaluated the histomorphological features and proposed a new scoring system for pathologic tumor response. The pathologic tumor response was categorized as complete response ( $p C R)$, no response ( $p N R)$, and partial response (pPR).

Results Of the total 50 patients, 2 patients had pCR, 3 had pNR, and 45 patients had PPR as per the new proposed scoring system. Note that $96 \%$ of the cases showed no disease progression.

Conclusion Bridge OMCT is a novel treatment method that can be used to tide over the waiting period between the diagnosis and surgery in resource-constrained institutions with heavy patient load. This mode of treatment in locally advanced OSCC seems to provide promising results in this setting. Large multicentric trials are warranted to confirm these results.
DOI https://doi.org/10.1055/s-0041-1735592 ISSN 2278-330X

How to cite this article: Aravind S, Jose J, Shenoy PK, et al. The Spectrum of Histomorphological Changes and Pathological Tumor Response following Preoperative Oral Metronomic Chemotherapy in Oral Squamous Cell Carcinoma South Asian J Cancer 2022;11 (2):146-151. (c) 2022. MedIntel Services Pvt Ltd. All rights reserved.

This is an open access article published by Thieme under the terms of the Creative Commons Attribution-NonDerivative-NonCommercial-License, permitting copying and reproduction so long as the original work is given appropriate credit. Contents may not be used for commercial purposes, or adapted, remixed, transformed or built upon. (https://creativecommons.org/licenses/by-nc-nd/ 4.0/)

Thieme Medical and Scientific Publishers Pvt. Ltd., A-12, 2nd Floor, Sector 2, Noida-201301 UP, India 


\section{Introduction}

Metronomic chemotherapy is a novel concept that involves frequent administration of chemotherapy drugs at doses below the maximum tolerated doses and with no prolonged drug-free break. Thus, a sustained low blood level of the drug without significant toxic side effects is achieved. ${ }^{1}$ The scientific basis for metronomic chemotherapy is that conventional antineoplastic drugs target vascular endothelial cell proliferation but the antiangiogenetic effect cannot be sustained as endothelial cells recover during treatment breaks and this may be overcome by frequent treatment at low doses. ${ }^{2}$ Oral metronomic chemotherapy (OMCT) is a multitargeted therapy that exerts its anticancer activity by inhibiting tumor angiogenesis, stimulating anticancer immune response, and inducing tumor dormancy. Thus, it exerts both direct and indirect effects on tumor cells and their microenvironment. ${ }^{3}$

More than 300 head and neck surgeries are performed at our institution every year. In view of the heavy patient load and constrained resources at our center, patients have a waiting period of 3 to 5 weeks from the diagnosis to definitive surgery and this causes additional psychological stress to the patients. With this background, bridge OMCT was practiced in stage $3 /$ stage 4 oral squamous cell carcinoma (OSCC) patients, so as to prevent disease progression during the waiting period. This protocol is being practiced in our center for the last 2 years and the patients for bridge OMCT are selected as per the institutional Multi-Speciality Board decision.

Even though there are a few reported studies which assessed clinically the efficacy and toxicity of preoperative OMCT, to the best of our knowledge, no study has been reported till date describing the spectrum of histopathological changes and pathological tumor response to bridge or preoperative OMCT in OSCC patients. There is no other feasible way to determine the positive effect of presurgical treatment than by histological examination of operative specimen.

The pathologists should be aware of the resultant morphological findings as bridge OMCT is likely to be used more frequently in the management of advanced stage head and neck cancer. In this study, we aimed to assess the spectrum of histomorphological changes and to propose a pathologic tumor response scoring following bridge OMCT in advanced stage OSCC. We believe that this study would also pave the way for future studies on exact mechanism of action of these drugs in preventing disease progression so as to develop specific biomarkers for predicting treatment response.

\section{Materials and Methods}

The present retrospective study was conducted on the surgical specimens of 50 OSCC patients received at the division of oncopathology of our institute. The study was approved by the Institutional Review Board (1616/IRB-SRC/ 13/MCC/13-04-2019/1). The inclusion criteria comprised of all patients with a diagnosis of stage $3 /$ stage 4 OSCC who had been treated with OMCT from June 2018 to May 2019 and had completed definitive surgery as per the Institutional Multi-Speciality Board decision. Patients with advanced stage OSCC who did not receive OMCT, those who had received preoperative conventional neoadjuvant chemotherapy (NACT), and those with recurrent oral cavity cancers were excluded. Initially, 54 patients were given OMCT regimen during the study period out of which 4 patients were exempted from surgery -3 patients had disease progression and were referred to palliative radiotherapy and 1 patient refused surgery due to personal reasons. Thus, total 50 patients were included in the present study. The OMCT schedule comprised of tablet methotrexate $15 \mathrm{mg} / \mathrm{m}^{2}$, administered once in a week, capsule celecoxib $200 \mathrm{mg}$ twice daily, and tablet erlotinib $100 \mathrm{mg}$ once daily "and the drugs were stopped" 2 weeks prior to surgery. The selected patients were assessed every 2 weeks for toxicities related to OMCT and for evidence of disease progression. In case of progression, patients were taken up for surgery as early as possible.

Baseline clinical data and treatment details were collected from case files and recorded in the pro forma.

Hematoxylin and eosin stained sections of formalin-fixed paraffin-embedded samples of tumor proper and metastatic lymph nodes were retrieved from archives of the division of pathology. Detailed histopathological examination was done for evaluating chemotherapy-induced changes. The tumor cells were evaluated for tumor differentiation, percentage of viable tumor cells, predominant and worst pattern of invasion (WPOI), nuclear-cytoplasmic ratio, mitotic count, and necrobiotic changes such as nucleocytoplasmic vacuolation, pyknosis, nuclear enlargement, and presence of nucleoli. The stroma was examined for host response including necrosis, fibrosis/collagenization, microcalcifications, acellular keratin, vascularity, and inflammatory cell infiltration. Perineural invasion and lymphovascular invasion were also assessed. Similar histomorphological changes, observed in the metastatic lymph nodes, were recorded.

Lymphocytic response in tumor tissue was graded and documented as:

Grade 1-dense infiltration of lymphocytes destroying tumor cells or forming masses.

Grade 2-formation of microaggregates of lymphocytes.

Grade 3-scattered lymphocytes between tumor cells.

As there is no universally accepted scoring system till date to assess the pathological response to OMCT therapy, we referred various accepted pathological response scoring systems for assessing NACT response in various organs. ${ }^{4}$ Finally, we formulated a new response scoring system on the basis of NACT response scoring criteria of the Japanese Head and Neck Society ${ }^{5}$ and scoring system by Hirakawa et $\mathrm{al}^{6}$ in head and neck SCC.

The tumor response was pathologically categorized as pathologic complete response ( $\mathrm{pCR}$ ) when no recognizable viable invasive tumor cells were present (0\%) and pathologic no response (pNR) when tumor did not exhibit any degenerative changes listed above (100\% viable tumor cells). Pathologic partial response (pPR) was defined as the presence of scattered individual or small clusters of tumor cells (viable 
Table 1 Tumor response scoring system developed as a part of the present study

\begin{tabular}{|l|l|l|}
\hline Category & Pathologic tumor response & Definition \\
\hline Grade 1 & $\begin{array}{l}\text { No effect/response } \\
(\mathrm{PNR})\end{array}$ & $\begin{array}{l}100 \% \text { viable tumor cells present with no cytological or histological } \\
\text { chemotherapeutic changes }\end{array}$ \\
\hline Grade 2a & Very minimal response (pPR) & $>70 \%$ of viable tumor cells \\
\hline Grade 2b & Mild response (pPR) & $40-70 \%$ of viable tumor cells \\
\hline Grade 2c & $\begin{array}{l}\text { Moderate response } \\
(\mathrm{pPR})\end{array}$ & $<40 \%$ of viable tumor cells \\
\hline Grade 3 & $\begin{array}{l}\text { Complete response } \\
(\mathrm{PCR})\end{array}$ & No recognizable viable tumor cells present $(0 \%)$ \\
\hline
\end{tabular}

and necrotic) in a desmoplastic or hyalinized stroma. The pPR category is further subdivided into very minimal ( $>70 \%$ of viable tumor cells), mild (40-70\% of viable tumor cells), and moderate ( $<40 \%$ of viable tumor cells) ( - Table 1 ).

The results obtained were interpreted and correlated statistically. The continuous data was expressed in terms of mean \pm standard deviation or median/interquartile range. The qualitative data was expressed in counts and percentage. Statistical analysis was done using SPSS software version 22

\section{Results}

\section{Clinical Profile}

Of the total 50 patients, 36 (72\%) were males. Age of patients who received bridge OMCT ranged from 34 to 73 years (mean: $53.76 \pm 10.88$ ). Most common primary tumor site was the lateral border of the tongue (34\%). Laterality was nearly equal with slight predominance noticed for the left side (52\%). On assessing pre-OMCT clinical staging, majority of the patients were grouped under cT4N1 (26\%) (American Joint Committee on Cancer 8th edition). Sixty-six percent of patients fell under T4 when grouping was based on T stage alone. Duration of OMCT ranged from 2 to 6 weeks with majority of patients (88\%) having completed 4 weeks of OMCT regimen. Forty-eight patients had taken three-drug combination of OMCT.

\section{Histopathological Profile}

Primary tumor: The histological examination of the primary tumor showed two cases with no viable tumor cells and three cases with $100 \%$ viable tumor cells present with no cytological or histological chemotherapeutic changes. Twenty-two cases (46\%) showed $>70 \%, 14$ cases (26\%) showed 40 to $70 \%$, and 9 cases (20\%) showed $<40 \%$ viable tumor cells ( - Fig. 1 ). The details of pathologic changes in tumor cells in postOMCT cases are shown in - Table 2 .

On assessing the distribution of WPOI, majority of the cases (52\%) showed type 5, 24\% cases showed type 3, while remaining $20 \%$ cases displayed type 4 pattern.

Pathologic changes in the stroma: Two cases (4\%) showed $>90 \%$ stromal necrosis, 1 case (2\%) showed > 90\% collagenization, and 32 cases (64\%) showed acellular keratin (-Table 3; -Fig. 2).
Inflammatory host response: Thirty-seven out of 50 cases showed lymphoplasmacytic, histiocytic, and giant cell type inflammatory host response around tumor cells. Ten cases showed lymphoplasmacytic infiltrate, 2 cases with mixed inflammation, and 1 case with purely histiocytic and giant cell type host response (-Figs. 1 and 3). Majority of cases (64\%) showed grade 1 lymphocytic host response, while $34 \%$ cases showed grade 2 and $2 \%$ showed grade 3 .

No significant difference was noted in tumor differentiation between pre- and post-OMCT cases with majority of the cases belonging to moderately differentiated/grade 2 OSCC ( $p$-value 0.856). Difference in post-OMCT tumor differentiation was

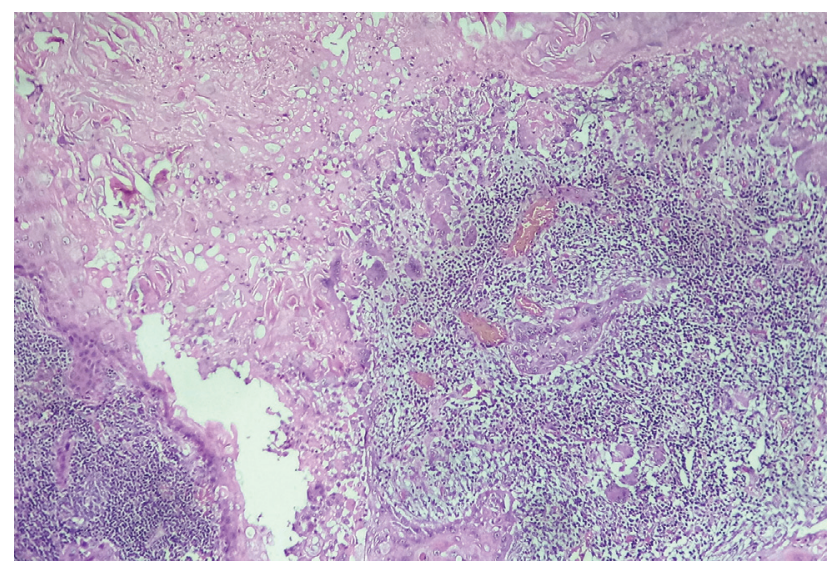

Fig. 1 Photomicrograph showing hematoxylin and eosin (H\&E) stained section of viable tumor cells with lymphohistiocytic reaction post-oral metronomic chemotherapy (OMCT) (40x).

Table 2 Pathologic changes in tumor cells in post-OMCT cases

\begin{tabular}{|l|l|}
\hline Pathologic changes in tumor cells & Frequency (\%) \\
\hline Increased N/C ratio & $48(96)$ \\
\hline Cytoplasmic vacuolation & $44(88)$ \\
\hline Nuclear vacuolation & $48(96)$ \\
\hline Pyknotic nuclei & $32(64)$ \\
\hline Nuclear enlargement & $47(94)$ \\
\hline Nucleoli & $48(96)$ \\
\hline
\end{tabular}

Abbreviations: N/C ratio, nuclear-cytoplasmic ratio; OMCT, oral metronomic chemotherapy. 
Table 3 Pathologic changes in the stroma of post-OMCT cases

\begin{tabular}{|l|l|}
\hline Pathologic changes in the stroma & Frequency (\%) \\
\hline Vascular hyalinization & $4(8)$ \\
\hline Microcalcification & $18(36)$ \\
\hline Acellular keratin & $32(64)$ \\
\hline PNI & $27(54)$ \\
\hline LVI & $2(4)$ \\
\hline Stromal collagenization & \\
\hline$<40 \%$ & $16(32)$ \\
\hline $40-90 \%$ & $33(66)$ \\
\hline$>90 \%$ & $1(2)$ \\
\hline Stromal necrosis & \\
\hline$>90 \%$ & $2(4)$ \\
\hline $40-90 \%$ & $14(28)$ \\
\hline$<40 \%$ & $21(42)$ \\
\hline $1-10 \%$ & $10(20)$ \\
\hline $0 \%$ & $3(6)$ \\
\hline
\end{tabular}

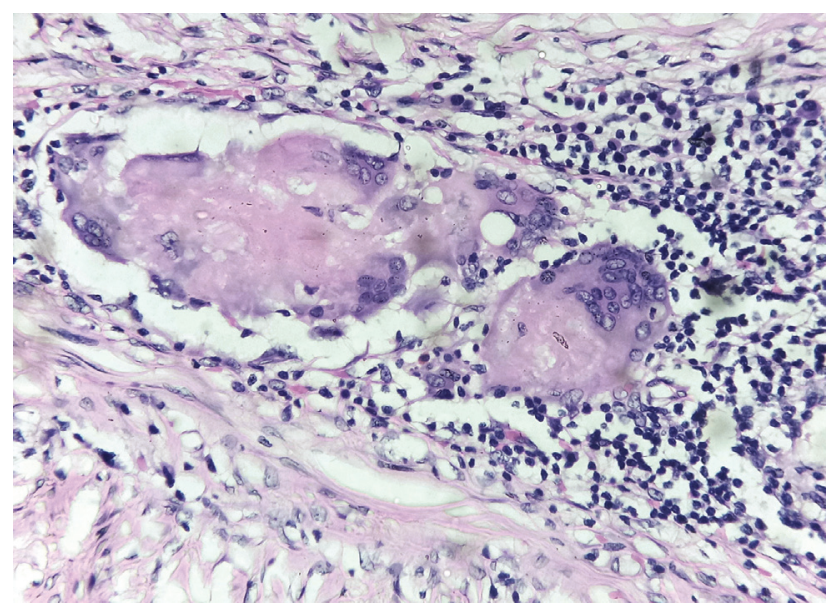

Fig. 3 Photomicrograph showing hematoxylin and eosin (H\&E) stained section with prominent giant cell reaction post oral metronomic chemotherapy (OMCT) (100x).
Abbreviations: LVI, lymphatic vascular invasion; OMCT, oral metronomic chemotherapy; PNI, perineural invasion.

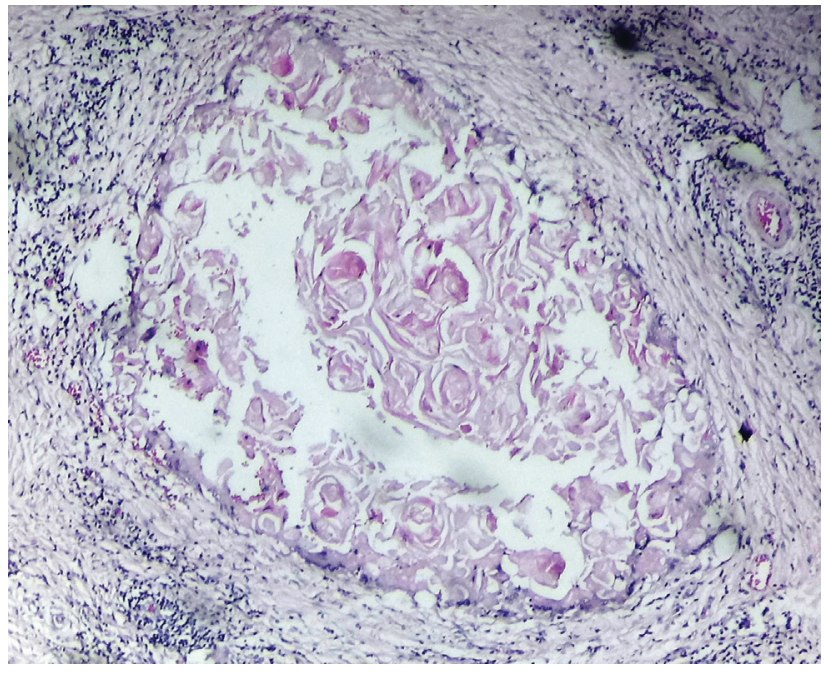

Fig. 2 Photomicrograph showing hematoxylin and eosin (H\&E) stained section acellular keratin post-oral metronomic chemotherapy (OMCT) (100x).

noted in 13 cases in which 7 cases showed better differentiation whereas 6 cases showed transformation into higher grade.

Pathologic changes in the metastatic lymph node: No viable tumor cells were seen in 17 cases (34\%), 42 cases (84\%) showed collagenization, $>90 \%$ necrosis was seen in $9(18 \%)$ cases, and acellular keratin in 25 cases (50\%) (-Table 4).

Pathologic tumor response at secondary site: Two cases (4\%) out of 50 showed pathologic complete response and 45 cases (90\%) showed partial response in which 22 showed minimal response, 14 showed mild response, and 9 showed moderate response.
Table 4. Pathologic changes in the metastatic lymph node

\begin{tabular}{|l|l|}
\hline $\begin{array}{l}\text { Pathologic changes in } \\
\text { the metastatic lymph node }\end{array}$ & Frequency (\%) \\
\hline$\%$ Viable tumor cells in lymph node & \\
\hline $0 \%$ & $17(34)$ \\
\hline$<90 \%$ & $18(36)$ \\
\hline$>90 \%$ & $15(30)$ \\
\hline$\%$ of nodal collagenization & \\
\hline$<40 \%$ & $42(84)$ \\
\hline $40-90 \%$ & $7(14)$ \\
\hline$>90 \%$ & $1(2)$ \\
\hline$\%$ nodal necrosis & \\
\hline$<40 \%$ & $34(68)$ \\
\hline $40-90 \%$ & $7(14)$ \\
\hline$>90 \%$ & $9(18)$ \\
\hline Microcalcification & \\
\hline Present & $11(22)$ \\
\hline Absent & $39(78)$ \\
\hline Acellular keratin & \\
\hline Present & $25(50)$ \\
\hline Absent & $25(50)$ \\
\hline Vascularity & \\
\hline Prominent & $17(34)$ \\
\hline Normal & $26(52)$ \\
\hline Decreased & $7(14)$ \\
\hline Extra nodal extension & \\
\hline Present & $20(40)$ \\
\hline Absent & $30(60)$ \\
\hline
\end{tabular}


Three patients showed no response to treatment. Of the nonresponders, two showed disease progression with upstaging from T3N2b to T4aN2c and T4bN0 to T4bN2c. The third case maintained the same stage of T4aN0.

In short, in response to bridge OMCT, $4 \%$ cases showed complete response, $60 \%$ cases has shown a stable disease, and $34 \%$ has shown tumor downstaging.

\section{Discussion}

Oral cancer is a major health care problem in rural India. Presentation at advanced stage, lack of adequate treatment facilities and manpower coupled with the lack of social security may contribute to suboptimal outcomes. A few studies are reported which assessed the efficacy of cetuximab-based OMCT in palliative settings in advanced stage head and neck cancers. ${ }^{3}$ Recent studies have shown that in resource-constrained institutions with heavy patient load and prolonged waiting period for surgeries, OMCT can be given to prevent disease progression (bridge OMCT) ${ }^{7}$

The bridge OMCT regimen used in our center composed of three drugs-methotrexate, erlotinib, and celecoxib. The combination of celecoxib, a cyclooxygenase-2 (COX2) inhibitor, and erlotinib, an epidermal growth factor receptor (EGFR) pathway blocker, has a biological rationale, as COX2 and EGFR pathways have significant crosstalk and resistance to EGFR tyrosine kinase inhibitor may be inhibited by COX2 inhibitor. Hence, combined inhibition can effectively target the tumor angiogenesis, apoptosis, tumor growth suppression, and decrease the metastatic potential. ${ }^{8,9}$

The duration of OMCT ranged from 2 to 6 weeks with majority of the patients (88\%) receiving it for 4 weeks. The drug celecoxib was omitted in two patients (4\%) who had a history of cardiovascular diseases.

In the present study, a spectrum of histopathological changes was observed in both tumor proper and metastatic lymph node with the use of bridge OMCT. The effects have been divided into two, as pathologic changes in tumor cells and changes in the stroma. Another salient feature focused was the presence of host inflammatory response.

In the present study, degenerative changes in cells such as necrosis, nuclear/cytoplasmic vacuolation, and pyknosis were noted along with features like nuclear enlargement, presence of nucleoli, and mitosis.

In a background of a large variety of retrogressive changes, the emphasis was to identify percentage of viable tumor cells, morphologically identified as cells with distinct nuclear chromatin with intact nuclear and cytoplasmic membrane in the absence of criteria of cell destruction (karyorrhexis, karyolysis, pyknosis). ${ }^{4}$

There was no significant difference in tumor differentiation between pre- and post-OMCT cases with majority of the cases belonging to moderately differentiated/grade 2 OSCC. Difference in tumor differentiation was noted in 13 cases in which 7 cases showed better differentiation after OMCT whereas 6 cases showed transformation into higher grade. Predominant pattern of invasion noted in most cases was type 3 and WPOI noted was type 5 based upon BrandweinGenseler scoring system. ${ }^{10}$

In the stroma, necrosis, fibrosis/collagenization, hyalinization of blood vessel, microcalcification, and neovascularization have been observed. Acellular keratin was another salient feature noted in $32(64 \%)$ cases. These features were previously described in surgical specimens with post-NACT in OCSCC, esophagus, breast, ovary, and colon. ${ }^{4,11-16}$

Prominent or increased stromal vascularity was noticed in 24 cases (48\%). This result is not consistent with previously published literature. ${ }^{17}$ Tumor endothelial cell and endothelial progenitor cell are the two major targets for the antiangiogenetic action of OMCT. Apart from toxicity to endothelial cells, OMCT also induces antiangiogenic protein thrombospondin-1, inhibits angiogenic hypoxia-inducible factor- $1 \alpha$, and decreases circulating vascular endothelial growth factor levels. ${ }^{17,18}$ As per this mechanism of action we expect a marked reduction in tumor vascularity, postOMCT.

Mpekris et al had developed a mathematical model that incorporates various proposed mechanisms, and reported that improved function of tumor vessels is a key determinant of benefit from OMCT. ${ }^{19}$ They found that OMCT induces functional normalization of tumor blood vessels, resulting in improved tumor perfusion. The improved vessel function enhances the delivery of oxygen and drugs, increases the number of effector immune cells, and decreases the number of regulatory $\mathrm{T}$ cells, which in turn kill a larger number of cancer cells, including cancer stem-like cells.

Literature search showed that the combination regimens can yield surprising outcomes and they may not always be more effective than single-drug therapy. A possible explanation of these findings is that the combined effects of chemotherapy on individual signaling pathways are additive, synergistic, or antagonistic, and depend not only on the oxidative antitumor agent/cytotoxic drug examined but also the dosages employed. ${ }^{20,21}$

Another interesting feature noticed was increased host inflammatory response which was mainly lymphocytic, histiocytic, and giant cell type. Foamy histiocytes and giant cells were seen around acellular keratin with islands of nonviable tumor cells. Similar observations are noted in many post-NACT pathologic response studies which may be indicative of host tissue response to necrobiotic tumor. ${ }^{4}$ A plausible explanation for this observation might be that the restoration and enhancement of the antitumor immune response is another aspect of metronomic regimens. OMCT selectively depletes Treg cells and thus restores the natural killer and $\mathrm{T}$ cell functions. ${ }^{22}$

Pathologically similar changes were observed in response to chemotherapy in metastatic lymph nodes but not as pronounced as in the primary site. Vascularity was not increased in metastatic lymph nodes as compared to tumor proper.

As there is no accepted scoring system till date to assess pathological response to bridge OMCT therapy, we referred various accepted pathological response scoring systems for NACT in various organs. Finally, we formulated a new 
response scoring system on the basis of post-NACT response scoring criteria published in the literature. ${ }^{5,6}$

Pathologic response of tumor proper alone was assessed based on percentage of viable tumor cells and stromal response such as necrosis, fibrosis, microcalcification, and acellular keratin.

The present study has shown that $4 \%$ cases showed complete response, $60 \%$ cases has shown a stable disease, and $34 \%$ has shown tumor downstaging which indicates that OMCT as bridge therapy is beneficial in buying time and preventing disease progression in resource-constrained settings. A largescale prospective study with sufficient power of selected cohort is mandatory to validate the utility of OMCT as bridge therapy in OSCC. Further studies are needed to obtain a vivid picture of the mechanism of action of OMCT drugs and thereby target on the development of potential biomarkers that would predict tumor response and progression.

\section{Conclusion}

It is mandatory for the pathologists and clinicians to be aware of the postchemotherapeutic morphological changes, as the histologic response to therapy or tumor response grade has been shown to correlate with the patient survival. Preoperative OMCT is a novel treatment method that can be used to tide over the waiting period between diagnosis and surgery in resource-constrained institutions with heavy patient load. Preoperative OMCT as a bridge in locally advanced OSCC seems to provide promising results in this setting. Large, multicentric trials are warranted to confirm these results and also for the development of specific biomarkers that can predict tumor response.

Pathological Tumor Response in Preoperative Oral Metronomic Chemotherapy in Oral Squamous Cell Carcinoma Aravind et al.

Funding

None.

\section{Conflict of Interest}

The authors declare that they have no competing interests.

\section{Acknowledgments}

None.

\section{References}

1 Bahl A, Bakhshi S. Metronomic chemotherapy in progressive pediatric malignancies: old drugs in new package. Indian J Pediatr 2012;79(12):1617-1622

2 Maiti R. Metronomic chemotherapy. J Pharmacol Pharmacother 2014;5(03):186-192

3 De Felice F, Musio D, Tombolini V. Head and neck cancer: metronomic chemotherapy. BMC Cancer 2015;15:677

4 Sethi D, Sen R, Parshad S, Khetarpal S, Garg M, Sen J. Histopathologic changes following neoadjuvant chemotherapy in various malignancies. Int J Appl Basic Med Res 2012;2(02):111-116
5 Hirakawa H, Hanai N, Suzuki H, et al. Prognostic importance of pathological response to neoadjuvant chemotherapy followed by definitive surgery in advanced oral squamous cell carcinoma. Jpn J Clin Oncol 2017;47(11):1038-1046

6 Hirakawa H, Hanai N, Ozawa T, et al. Prognostic impact of pathological response to neoadjuvant chemotherapy followed by definitive surgery in sinonasal squamous cell carcinoma. Head Neck 2016;38(Suppl 1):E1305-E1311

7 Pai PS, Vaidya AD, Prabhash K, Banavali SD. Oral metronomic scheduling of anticancer therapy-based treatment compared to existing standard of care in locally advanced oral squamous cell cancers: a matched-pair analysis. Indian J Cancer 2013;50(02): 135-141

8 Shin DM, Zhang H, Saba NF, et al. Chemoprevention of head and neck cancer by simultaneous blocking of epidermal growth factor receptor and cyclooxygenase-2 signaling pathways: preclinical and clinical studies. Clin Cancer Res 2013;19(05):1244-1256

9 Patil VM, Chakraborty S, Jithin TK, et al. An audit of the results of a triplet metronomic chemotherapy regimen incorporating a tyrosine kinase inhibitor in recurrent/metastatic head and neck cancers patients. South Asian J Cancer 2016;5(02):48-51

10 Brandwein-Gensler M, Teixeira MS, Lewis CM, et al. Oral squamous cell carcinoma: histologic risk assessment, but not margin status, is strongly predictive of local disease-free and overall survival. Am J Surg Pathol 2005;29(02):167-178

11 Braun OM, Neumeister B, Popp W, et al. Histologic tumor regression grades in squamous cell carcinoma of the head and neck after preoperative radiochemotherapy. Cancer 1989;63(06):1097-1100

12 Mandard AM, Dalibard F, Mandard JC, et al. Pathological assessment of tumor regression after preoperative chemoradiotherapy of esophageal carcinoma. Clinicopathologic correlations. Cancer 1994;73:2680-2686

13 O'Neil M, Damjanov I. Histopathology of colorectal cancer after neoadjuvant chemoradiation therapy. Open Pathol J 2009; 3:91-98

14 Chakrabarti S, Mandal PK, Chowdhury AR, Das S. Consequence of neo-adjuvant chemotherapy on morphology of breast carcinoma: a systematic evaluation. Indian J Cancer 2016;53(01):29-33

15 McCluggage WG, Lyness RW, Atkinson RJ, et al. Morphological effects of chemotherapy on ovarian carcinoma. J Clin Pathol 2002; 55(01):27-31

16 Wedemeyer I, Kreppel M, Scheer M, Zöller JE, Büttner R, Drebber U. . Histopathological assessment of tumour regression, nodalstage and status of resection margins determines prognosis in patients with oral squamous cell carcinoma treated with neoadjuvant radiochemotherapy. Oral Dis 2014;20:e81-e89

17 Simsek C, Esin E, Yalcin S. Metronomic chemotherapy: a systematic review of the literature and clinical experience. J Oncol 2019; 2019:5483791

18 Marçola M, Rodrigues CE. Endothelial progenitor cells in tumor angiogenesis: another brick in the wall. Stem Cells Int 2015;2015 (02):832649

19 Mpekris F, Baish JW, Stylianopoulos T, Jain RK. Role of vascular normalization in benefit from metronomic chemotherapy. Proc Natl Acad Sci U S A 2017;114(08):1994-1999

20 Norrby K. Metronomic chemotherapy and anti-angiogenesis: can upgraded pre-clinical assays improve clinical trials aimed at controlling tumor growth? APMIS 2014;122(07):565-579

21 Martindale JL, Holbrook NJ. Cellular response to oxidative stress: signaling for suicide and survival. J Cell Physiol 2002;192(01): 1-15

22 Ghiringhelli F, Menard C, Puig PE, et al. Metronomic cyclophosphamide regimen selectively depletes $\mathrm{CD} 4+\mathrm{CD} 25+$ regulatory $\mathrm{T}$ cells and restores $\mathrm{T}$ and NK effector functions in end stage cancer patients. Cancer Immunol Immunother 2007;56(05): 641-648 